\title{
Pluralismo moral y lugares de la argumentación
}

\author{
M. ${ }^{\mathrm{a}} \mathrm{T}$. LÓPEZ DE LA VIEJA \\ Facultad de Filosofia \\ Universidad de Salamanca
}

«... el Liberalismo político intenta responder a la pregunta: icómo es posible que llegue a existir una sociedad estable y justa en la cual los ciudadanos, libres e iguales, estén profundamente divididos por doctrinas religiosas, filosóficas, morales...? (J. Rawls, 1993, p. 133). La pregunta de Rawls se refiere a un tipo de dificultades que afectan a las sociedades contemporáneas, por ser sociedades pluralistas. ¿Cómo construir un marco de acción cooperativa a partir de posiciones o creencias distintas, opuestas incluso? El «hecho del pluralismo» - como J. Rawls to denomina- tiene consecuencias relevantes, tanto para el punto de vista moral como para la organización política. Entre otras cosas significa que existen, que deben coexistir en un mismo espacio, diferentes ideas del bien, formas de vida, ideologías, maneras de organizar las actividades y de entender el mundo, etc. ¿Cómo conseguir una «sociedad bien ordenadaw contando con este dato, la multiplicidad de creencias y de visiones del mundo? En principio, esta nueva situación o "hecho del pluralismo* muestra la apertura de las sociedades liberales: los individuos pueden asumir aquellas creencias y doctrinas que consideren razonables. El principio de tolcrancia sirve precisamente para orientar la acción en este nuevo contexto plural.

La ausencia de sistemas omnicomprensivos no es, pues, un serio inconveniente para establecer las bases de una cultura pública. En el marco de las democracias constitucionales, el «hecho del pluralismo» se presenta como una oportunidad para definir relaciones más abiertas, y para cvitar actitudes dogmáticas, intolerantes o fanáticas. Sin embargo, icó́mo argumentar sobre cuestiones prácticas, en ausencia de tales sistemas omnicomprensivos? Tanto J. Rawls (1985) como J. Habermas (1996, pp. 277-292, 1998), R. Goodin (1989, pp. 1-8) y J. Raz (1986, pp. 110-133), por citar sólo algunos autores, han explicado a lo largo de los últimos años por qué no pueden coincidir los principios de la Filosofía moral con formas de vida concretas, ni con el contenido de una determinada idea del bien. El Liberalismo es, pues, una teoría antiperfeccionista. Esto explica, en parte, el marcado procedimentalismo, e incluso formalismo de algunos programas de la Filosofía práctica actual. Los procedimientos, la forma de argumentar, tienen que tratar de alguna manera la fragmentación, la inconmensurabilidad, la pluralidad, la heterogeneidad, etc., aspecto al cual me voy a referir aquí. La argumentación, irequiere de algún tipo de punto de partida, premisas anteriores a las reglas de procedimiento propiamente dicho? $\mathrm{O}$, lo que es lo mismo, una noción fuerte y amplia de «lo común" parece innecesaria, es más, su reivindicación puede crear serios inconvenientes para ciudadanos con derechos y libertades, y con ideas propias sobre el bien. Sin embargo, esto plantea otra cuestión, de índole diferente y menor, tal vez, referente a un punto de partida, parecido o común para los agentes que deliberan y argumentan sobre temas que les conciemen a todos, como ciudadanos. De un lado, es posible deshacerse de doc- 
trinas con pretensiones fuertes, o de visiones del mundo que no contemplan alternativas o excepciones pero, de otro lado, mayor pluralismo implica también defínir ciertos lugares para la argumentación, como punto de partida para el debate sobre cuestiones que interesan a todos o a la mayoría de los ciudadanos.

Los «lugares comunes» y su funcionalidad eran conocidos desde antiguo (R. Barthes, 1970); Aristóteles se ocupó de los topoi; por lo loci communes se interesaron despuếs Quintiliano, Cicerón $\mathrm{y}$, mucho después, G. Vico. Pero no, no se trata de recuperar la Tópica clásica -que correspondía a una forma de vida, ya olvidada (M. T. López de la Vieja, 1994, pp. 153-164)-, sino de considerar si hacen falta los «lugares comunes» en un contexto de argumentación donde tienen prioridad la diferencia y la heterogeneidad. Digamos que estas preguntas pueden ser abordadas desde una teoría de la argumentación, como Tópica formal. ¿Qué tipo de información compartida es necesaria para iniciar un razonamiento? Los «lugares» $n$ topoi cumplian esta función, la de crear una opinión común -Aristóteles los denominaba «argumentos ya dispuestos»-; la Tópica era parte de la Retórica, recuperada parcialmente por la Filosofía del Derecho en los años cincuenta y sesenta (Ch. Perelman, 1979). No obstante, la Tópica actual —que es la que aquí realmente interesa - dista mucho de la antigua noción $\mathrm{y}$ de las antiguas expcctativas (Th. Viehweg, 1991, 1986). Hoy tiene que definir de nuevo su papel, pues el corpus doctrinal con el cual entroncaba, la Retórica, pertenece por completo al pasado y a formas de vida que no pueden ni deben reconstituirse. Por lo tanto, hablar una vez más de «tópicos», o de «lugares comunes», tienc un sentido distinto, opuesto, tal vez, al sentido que se les atribuyó en la Tópica clásica: frente al uso dogmático, ahora puede tener un sentido crítico. La idea de klugares de la argumentación" alude ese papel alternativo que pueden ocupar los tópicos, aportando información sobre por qué y con qué objetivos entramos en procedimientos argumentativos. Los «lugares» dicen el qué, quiénes y el para qué de los argumentos. Pero no van más allá, ni tienen contenido normativo alguno. Parece importante subrayarlo desde un principio, pues esto marca las distancias con respecto a otros posibles usos de los tópicos, no críticos.

El punto de vista asumido aquí tiene que ver más bien con una «Tópica crítica» o «Tópica formal», tal como plantean Kopperschmidt (1973, 1981, 1985), Gethmann (1979, 1980) y Alexy $(1995,1991,1989)$, por ejemplo: 1) El punto de partida será el citado "hecho del pluralismo»; 2) para plantear la hipótesis de que los «lugares comunes» tienen un nuevo sentido en el marco de la «democracia deliberativa», 3) por tanto, se puede sugerir un uso alternativo, crítico, de los argumentonum sedes, mostrando en qué sentido amplían la información requerida para debatír en la esfera pública. Informan de algo que es relevante como, por ejemplo, los mecanismos de exclusión que operan en las relaciones sociales y políticas. En fin, los «topoi» preparan una opinión, crean las condiciones de aceptabilidad y, casi siempre, indican el rastro profundo - a veces no evidenteque han dejado los discursos de desigualdad y asimetría. Por usar un término que, en otro contexto teórico, emplea $C$. Sunstein $(1993,10,68-92,992,1991)$ : los lugares de la argumentación definen la «línea de base», el tranfondo injusto, el status quo que plantea algunos problemas para el modelo liberal, de justicia, igualdad y tolerancia.

\section{Pluralismo}

J. Rawls (1993, 1985) ha admitido que su noción de "sociedad bien ordenada», esto es, la sociedad que acepta el principio de 
justicia, no era una idea realista. El «hecho del pluralismo" corresponde a una situación en la cual se encuentran, por lo general, las sociedades democráticas modernas. Al centrar su análisis en las democracias constitucionales, J. Rawls pretende distanciarse en parte de las controversias filosóficas, ocupándose más bien de la estructura básica en estos sistemas, los sistemas democráticos: los agentes racionales, o quienes les pueden representar, quienes construyen una cultura pública sobre bases apropiadas, de justicia como equidad. Las sociedades modemas se caracterizan, como sabemos, por su pluralismo, por la existencia de doctrinas distintas en lo religioso, lo filosófico, lo moral. Todas ellas tienen cabida, han de tener cabida en la sociedad, siempre que se trate de doctrinas razonables; corresponde entonces a las instituciones proteger la libertad y, por tanto, la pluralidad de tales doctrinas. En esta circunstancia, ninguna concepción de justicia podrá considerarse más apropiada que otras, ninguna será superior o más comprensiva que las demás.

El Liberalismo político no se identifica, en fin, con ningún ideal particular $y$, por tanto, tampoco contempla la posibilidad de un sistema omnicomprensivo. De una parte, la cultura democrática garantiza que todas las doctrinas y concepciones pueden ser aceptadas. En la medida en que cumplan con los requisitos de la racionalidad. Cada persona moral puede formular, sin restricciones, su propio proyecto de vida buena. Rawls se interesa, en suma, por una sociedad estable, una sociedad de ciudadanos libres e iguales, en la cual sólo cabe pensar en un tipo de consenso por «solapamientom. Su objetivo es la cooperación, pero sin la necesidad de acudir a una doctrina filosófica -o religiosa- comprensiva. En los últimos años, este autor se ha centrado en los rasgos y consecuencias de este ahecho del pluralismo", eludiendo con esto complicadas discusiones sobre los proyectos personales de vida y sobre el valor de las creencias.

La solución procedimental es compartida por otros autores, si bien las conclusiones sobre el pluralismo y sus consecuencias son bastante diferentes. Por ejemplo, J. Clayton (1993, VII-IX) cree que todavía no se ha entendido bien qué significan esta diversidad y el abandono de un enfoque monolítico para el crecimiento de las sociedades multiculturales, sensibles como nunca a los "placeres de Babel", En cambio, el liberal J. Kekes $(1992,1993)$ teme que los cambios profundos, que ya se han producido, afcetarán en modo duradero a la fortaleza de la moralidad misma. Constata asimismo los desacuerdos radicales, concluyendo que no hay garantía racional para saber claramente qué está bien y qué está mal. En su opinión, el pluralismo ha llevado las dificultades hasta el centro mismo de la moralidad. Si no puede haber un solo sistema de valores, habrá que distinguir entre valores primarios y secundarios, etc. Es decir, el conflicto y la inconmensurabilidad aparecen como resultado del pluralismo. Entre los elogios hacia la pluralidad y las críticas de este tipo, que temen por la solidez de los principios y de los valores morales, digamos que es cierto, el pluralismo crea algunas dificultades. Pero también ofrece recursos para resolverlas de forma razonable, en un marco de derechos y libertades para los ciudadanos.

La Filosofía práctica ha entrado en una etapa parccida, de reflexión sobre el significado de pluralismo y debilidad de los sistemas fuertes. Esta apertura favorece la discusión interna $y$, de alguna manera, favorece también las soluciones formales antes que las propuestas sustantivas. A sabiendas, tambiên, de que, al final, los procedimentos no son demasiado explícitos sobre qué pasos conducirán a los agentes racionales hasta acuerdos razonables. El formalismo y procedimentalismo tienen ese inconvemiente, señalado en numerosas ocasiones. La Filosofía política parece 
seguir un desarrollo paralelo, hacia mayor apertura; así C. Leggewie (1994, pp. 3-18) se ha referido a una fuzzy Politics, es decir, un marco más flexible, resultado del descrédito en que habían caido los fundamentos más rígidos y dogmáticos. Dc hecho, las teorías liberales proponen restringir las funciones del Estado, aunque, al mismo tiempo, ponen de manifiesto el alcance real de los desacuerdos, lejos de un hipotético acuerdo universal. En esta misma línea, las teorías de la democracia moderna adoptan los principios del individualismo, sustituyendo el criterio de unanimidad por el acuerdo de las mayorías en la toma de decisiones. Por lo tanto, la legitimidad del sistema no tiene por qué descansar sobre la unanimidad. En realidad, se trata de dos soluciones paralelas: restricción procedimental en las teorias, propuestas o programas restringidos (thin) (M. Walzer, 1994, IX-XI) para abordar las cuestiones prácticas.

\section{Lugares de la argumentación}

En la búsqueda de decisiones cooperativas aparecen asimismo dos modelos de democracia: «estratégico» y «deliberativo», El modelo «estratégico»-por lo menos en la definición de D. M. Estlund (1993)adopta el tipo de racionalidad instrumental. El individuo racional trata de promover sus propios fines, de modo que el criterio fundamental no puede ser otro que la eficiencia. Por el contrario, el modelo «deliberativo" (J. Cohen, 1986, 1991; S. Benhabib, 1994) se aparta de la versión estratégica o económica de la democracia. En lugar de atender a los motivos individuales, se ocupa de la cooperación social. ¿Por qué medios? El sistema equitativo de cooperación descansa sobre el empleo de la razón. De ahí que el intercambio de argumentos, la deliberación, el juicio terminan por sustituir los estándares más objetivos por la formación de acuerdos racionales.
A falta de criterio definitivo sobre la verdad o falsedad en el ámbito práctico, la organización política sigue el criterio siguiente: un sistema o una decisión son correctos si se encuentran más allá de las objeciones razonables. En definitiva, la deliberación significa elegir entre distintas alternativas. Pese a estar asentada en un método no perfecto, la teoría de la democracia como deliberación propone un resultado razonable, a partir de un proceso de toma de decisiones que estará más allá - o más acá- de la unanimidad.

La democracia deliberativa interesa a aquellas teorías que, por lo general, comparten dos rasgos: prioridad de los procedimientos y concepción epistémica de la democracia. Ambos rasgos se dan de forma destacable en la teoría de J. Habermas (1994), por citar un ejemplo. Su versión procedimental de la democracia amplia un punto de vista que ya había expuesto a propósito de la Moral, definida en términos de formalismo, teoría deontológica, universalista y postconvencional. I a prioridad de los mejores argumentos como criterio no cambia: las decisiones en el ámbito público descansan sobre el uso de la razón, buenos argumentos. Se refiere en este caso a procesos institucionalizados de deliberación y para la toma de decisiones, para conseguir resultados tan racionales como equitativos. Al igual que J. Habermas, otros autores - J. Cohen, S. Benhabib, Ch. Mouffe, P. Weithman, M. Warrenencuentran en la «racionalidad deliberativa» la metodología más apta para las decisiones que tienen lugar en el marco de las sociedades modernas, democráticas. Pues se trata asimismo de sociedades pluralistas, heterogéneas, fragmentarias, descentradas y complejas.

Llegados a este punto, parece evidente que los procedimientos argumentativos ocupan, cada vez más, una posición central en la Filosofía práctica. En la teoría de la democracia, el uprocedimentalismo epistémico' (J. Cohen, 1986) implica que 
la actividad de votar se asemeja bastante a los juicios epistémicos. Por este motivo, la democracia no está definida, no necesita ser definida en términos de alguna comunidad determinada ni según una forma de vida concreta. En la democracia como procedimiento, una vez más, el papel más destacado corresponde a las «buenas razones», a la argumentación. Pues son el único medio para la obtención de acuerdos, sin la interferencia de cualquier tipo de dominación, pertenencia a tradición o cultura. Así pues, las razones ocupan un lugar indiscutible en los sistemas que exigen debate, deliberación pública. Sin embargo, esta solución no es tan sencilla como parece, ¿qué es o qué se entiendc por un buen argumento? Existen aquí algunos aspectos que vale la pena analizar, pues afectan a esa pieza fundamental de las teorías deliberativas, el razonamiento: 1) El procedimentalismo epistémico no suele distinguir entre el sentido débil y el sentido fuerte de la «buena» argumentación; tampoco matiza qué es argumento y qué argumentación; 2) además, prefiere la argumentación ideal a la argumentación fáctica (R. Alexy, 1995); 3) tampoco considera suficientemente el hecho de que toda discusión tiene un punto de partida. Se trata, por tanto, de tres dimensiones a tener en cuenta, significado, pragmática, tópica de la argumentación, para estar en situación de afirmar que, ahora más que nunca, es necesario revalorizar la argumentación. ¿Qué se entiende por «argumentar»?

1. Según A. Goldman (1994), el sentido débil de kbucn» argumento se refiere a una conclusión que se sigue de ciertas premisas. En cambio, en su sentido fuerte cl buen argumento contará con premisas verdaderas. Esto lleva a otro aspecto del «buen» argumento: una consideración abstracta del mismo no es lo mismo que el argumento como acto de habla complejo. De manera que las nomas de una «buena argumentación», en sentido fuerte, remi- ten a la situación epistémica de los interlocutores: estándares tácitos, creencias verdaderas, reglas vinculadas a la propia cultura, etc. Los argumentos sirven para obtener acuerdos, pero, frente a las teorías del consenso puro, otras versiones asumen que la situaciốn epistémica incide también en los factores sociales y motivacionales de la argumentación. $Y$ asi se justifica la tipología introducida hace ya un tiempo por W. Klein (1980): «argumentación» corresponde a una estructura abstracta, un acto de habla complejo, mientras que «argumentar» se refiere a la actividad de los discursos fácticos, dentro de un contexto de acción y como algo que ocurre en un espacio y en un tiempo. Esto quiere decir, que la Lógica de la argumentación no es coextensiva con la Pragmática del argumentar, en la cual aparecerá información relevante de ese segundo tipo.

2. ${ }^{\circ}$ El procedimentalismo epistémico se ha interesado por la estructura de los argumentos, como producción lingüistica compleja; pero apenas o nada por el análisis de las argumentaciones, en tanto acontecimientos espacio-temporales. ¿Por qué razón? La "concepción epistémica", que ya hemos encontrado en el caso de la teoría de la democracia deliberativa, suele preferir la argumentación ideal a la argumentación fáctica. La primera permite atender a las reglas del discurso, la segunda, la argumentación fáctica, se refiere a contextos informales, a acciones cotidianas y a contextos institucionales, como explica el mismo Klein. Es decir, el contexto comunicativo no forma parte del procedimiento, en caso de que éste haya sido pensado para garantizar la correción de las normas. Sólo - o nada menos- para la corrección de las normas.

3. ${ }^{\circ}$ R. Alexy $(1995,165-174,1989)$ analiza la situación de la teoría discursiva, para mostrar que, en efecto, la versión procedimental más estricta dice que las normas (N) son resultado del procedimiento (P). Y que el acuerdo descansa en el segui- 
miento de reglas. Pero - éste es el problema - cl procedimiento no llega a definir las operaciones que permitirán llegar al resultado. Por tanto, el discurso o argumentación «ideal» supone condiciones tales como tiempo indefinido, participantes en número ilimitado, ausencia de constricciones, etc. Condiciones irreales. Según esto, las reglas del discurso y la corrección procedimental forman parte de una idea meramente regulativa de argumentación. En cambio, los «argumentos» se sitúan a otro nivel, ya que tienen que ver con algo diferente, con la situación de los participantes y con las condiciones de aplicabilidad. De lo cual se deduce que la Pragmática de la argumentación tendrá todavía algo que decir, junto a la Lógica de la argumentación.

La situación epistémica y las limitaciones de la argumentación fáctica -en las que se detenia R. Alexy- ponen en evidencia las limitaciones de procedimientos que sólo sean «buenos» argumentos, pero no argumentaciones, en el segundo sentido. Es cierto que las teorias más formalistas -...como la teoría discursiva - están comprometidas con un modelo ideal. Sin. embargo, las argumentaciones y el contexto comunicativo muestran que el procedimentalismo no es suficiente en algunos casos o para algún tipo de funciones. $\mathrm{La}$ necesidad de ir más allá produce lo que R. Alexy denomina «corrección procedimental relativa». Esto es, el ajuste entre la idea meramente regulativa $y$, en el otro extremo, la inseguridad introducida por las personas reales y los acuerdos fácticos. R. Alexy admite que las proposiciones normativas no se confunden ni reducen a expresión de actitudes o sentimientos. Pues la corrección de las proposiciones normativas forma parte únicamente de la justificación interna. Si se desea algo más, contar con otro tipo de justificación - de tipo externo- junto a las reglas del discurso habrá que aceptar el grado de relatividad que introducen los participantes fácticos y, sobre todo, el tipo de convicciones fácticas que preceden $\mathrm{y}$, a veces, motivan la argumentación. Toda discusión ticne un punto de partida, ha de empezar por algo; Alexy distingue entonces entre la estructura racional y las epremisas iniciales», que sí tienen un contenido (1995).

La Tópica se ocupa de esto. Aristóteles se refíió a los «argumentos ya dispucstos» y a los topoi o conceptos comunes. Quintiliano los denominaba umanantiales de donde debemos sacar las pruebas". G. B. Vico creía en un fondo público y compartido de verdad. Ch. Perelman (1979), ya en la etapa de Nueva Retórica, afirma que los lugares comunes juegan en la argumentación un papel análogo al de los axiomas en un sistema formal. Pero, entre tanto, se han perdido por completo las condiciones para una Tópica material, por haber estado unida a un sistema, en el cual el sentido común estaba ligado a una forma de vida, mucho más homogénea que las actuales formas de vida, plurales y heterogéneas. El contexto de pluralismo -al que se refiere J. Rawls (1993) - convierte en anacrónicas a las soluciones que vuelvan al pasado, una vez anulada la posibilidad de un fondo común de opiniones. Entonces ¿́por qué y para qué la Tópica? La Tópica formal no es igual que la Tópica clásica. Por lo mismo, tampoco vuelve hacia formas de vida compartidas: las tradiciones y el marco general que sustentó las prácticas de la Retórica clásica se han disuelto por muchos y buenos motivos. Sin embargo, en un nivel más restringido, la argumentación práctica no sólo necesita reglas, también requiere de alguna información relevante. La información que está en los lugares o premisas iniciales.

\section{El uso critico de los lugares comunes}

¿Existe un uso crítico de la Tópica formal? La tradición clásica asociaba los argumentorum sedes con una asunción no crítica 
de las creencias y, por tanto, los tópicos eran siempre vulnerables ante el cngaño y ante la manipulación. El empleo sistemático de los tópicos no sólo era deficiente desde el punto de vista cognitivo, sino que, aún más, era parte de una falsa conciencia, con desfavorables consecuencias prácticas. Por tanto, los tópicos cumplían una función ideológica, en el peor de los casos, pues trabajaban en contra de cualquier interés emancipatorio $y$, en el mejor de los casos se convertian en superfluos o carentes de significado. La consideración actual de la Tópica ha cambiado, como, en general, la valoración de la Retórica; a partir del ugiro retórico» que tiene lugar desde los años sesenta y setenta en la Filosofía prâctica. No existen, en efecto, muchos «lugares" que sean realmente comunes en las sociedades multiculturales y pluralistas, ni tiene por qué haberlos. Al menos desde una perspectiva antiperfeccionista, como la que suclen adoptar las teorías liberales.

¿Cómo entender ahora el papel de la Tópica formal? Desde una teoría de la argumentación. El análisis de las argumentaciones fácticas aporta información relevante sobre relaciones prácticas, e incluso sobre el peor aspecto de las sociedades modernas, liberales y pluralistas: las relaciones de dominio. Dominación que genera, claro está, desacuerdos profundos, destruyendo sistemáticamente las posibilidades de cooperación. La búsqueda de acuerdos sin interferencias del dominio induce a entrar en procedimientos argumentativos, en general. Ahora bien, esto implica también entrar en otro nivel de los argumentos: preguntar menos por las condiciones generales de una cultura pública, preguntar más por la situación de los agentes, sus relaciones, el contexto real. Si se acepta esta importante restricción, el uso crítico de la Tópica formal tiene que ver sobrc todo con dos objetivos, no menos interesantes que los anteriores: 1) tener información sobre las condiciones reales de la comprensión intersubjetiva, estableciendo premisas plausibles; 2) definir las condiciones institucionales, prácticas, de la argumentación:

1) El uso crítico de la Tópica arrancará, pues, con el repertorio de estereotipos, comunidades de creencia, temas aceptados, aquellos que circulan habitualmente a través de la opinión pública, etc. En lugar de considerarlos restos de un uso no racional, poco interesante, se puede extraer su contenido cognitivo $\mathrm{y}$, gracias a esto, llegar a un uso crítico. Cuando se acepta su relevancia cognitiva, los lugares comunes se convierten en argumentonum sedes. Es decir, recobran su valor heuristico. J. Kopperschmidt $(1973,1985)$, quien se ha interesado por las nuevas funciones de la Retórica, ha defendido también esto, que los valores y creencias son funcionales desde el punto de vista argumentativo, ya que forman parte de la búsqueda de premisas. Pero hay algo más que premisas plausibles. Este método o técnica de pensamiento -así definía Th. Viehweg a la Tópica - forma parte de una versión complementaria de la Lógica, aquello que S. Toulmin (1958) llamó «Lógica aplicable» y C. T. Gethmann (1979) «Protológica». La construcción argumentativa aproxima entonces Lógica y Retórica, pues los argumentos tienen una secuencia y una historia, aparecen como lo que son en realidad: argumentos en el tiempo.

2) Por lo tanto, el análisis sistemático de las argumentaciones fácticas aporta información sobre la situación epistémica. El uso crítico de la Tópica puede incluir otras funciones, además de introducir premisas plausibles. Para terminar, sirvan un par de ejemplos para mostrar por qué importa identificar correctamente dónde está la facticidad de los argumentos o la "línea de partida», "línea de base», como la denomina C. Sunstein (1992, 1993):

2.1. ¿Puede hablarse de «neutralidad» liberal? Las sociedades liberales exigen 
imparcialidad, cierto grado de neutralidad también. Pero llegan con frecuencia a resultados opuestos, parciales. Por esta razón, un conocimiento pormenorizado del status quo, de cómo son realmente las relaciones y cómo está la situación de los agentes, evita que, en nombre de la neutralidad, los resultados de la acción política sean incorrectos, parciales o tendenciosos. Sólo el conocimiento de las distribuciones realmente existentes -como sostiene C. Sunstein-permite que la intervención de las instituciones o del Estado, teóricamente imparcial o neutral, no derive finalmente hacia una parcialidad injustificable, a favor de quienes previamente han sido beneficiarios en la distribución de bienes o de oportunidades. Los programas de acción afirmativa - que tantas resistencias provocan en quienes han disfrutado de la mayoria de los privilegios- ejemplifican lo anterior, cómo equilibrar las posiciones entre mayorias y minorías, o de quienes, por pertenencia a un género, por poner un caso claro, no pueden quebrar un estado de cosas, una «línea de base». En el marco de una sociedad liberal, con pretensiones de justicia, igualdad, tolerancia.

2.2. En una cultura profundamente antirretórica como la actual, el giro hacia la Tópica ha de justificar claramente su aportación heurística y crítica. Otro ejemplo más: el Fascismo (K. Ehlich, 1989) cra también una forma de discurso. Un discurso en el cual el tópico de la «lengua materna* sirvió para construir un sistema de exclusión y, al final, un sistema de clasificación y de exclusión. En lenguaje se transformo paso a paso en un campo de batalla, como los hechos históricos demuestran. Aún lo es, un campo de tensiones. Sobre todo alli donde subsisten las modalidades que sirven para desacreditar, minusvalorar o para introducir la violencia en la vida cotidiana. Asi han sido denunciadas por la Crítica feminista ( $\mathrm{S}$. Trömel-Plötz, 1994). Lo sigue siendo allí donde la integración de minorías étnicas exige la gravosa asunción de un «rol modelico» (R. Delgado, 1991) que, al final, resulta ser un papel distorsionador, profundamente falaz para los protagonistas de las situaciones de desventaja o discriminación.

La información sobre casos concretos ayuda, pues, a entender la diferencia entre «buenos» argumentos y argumentos que son formalmente correctos. Desde un punto de vista interno. No desde un punto de vista externo (J. Wroblewski, 1989). La pregunta sobre cómo llegar a sistemas que faciliten la cooperación, partiendo de la diversidad y la pluralidad que son valiosas $y$, por tanto, irrenunciables, llevaba al principio de tolerancia (J. Rawls, 1993). Aceptar la pluralidad como algo valioso implica, además, retener información sobre cómo se experimentan y se realizan esas formas de vida diferentes. En la práctica, cuentan, pues, los procedimientos, pero algunas veces cuenta también la situación fáctica de quienes argumentan en una situación deteminada. Y desde ahí hay que comenzar. Las premisas, lugares comunes -relativamente comunes- sirven de punto de partida o a titulo de premisas argumentales. Los topoi han cambiado de papel y de contexto, no han desaparecido, ya que todavía preparan, crean las condiciones para aceptar algo sin demasiado esfuerzo crítico. Por eso, ofrecen información sobre la pauta real que siguen los discursos y las relaciones fácticas: el whecho del pluralismo" no siempre es o todavía no es un hecho de justicia, de igualdad $y$ de tolerancia.

\section{REFERENCIAS BIBLIOGRÁFICAS}

AlEXY, R. (1995): Recht, Vemunft, Diskurs, Frankfurt, Suhrkamp.

- (1991): «Idee und Struktur eines vernünftigen Rechtssystems", ARSP, núm. 44, pp. 30-44.

- (1989): «Probleme der Diskurstheorie», Zeitschrift für philosophische Forschung, núm. 43 , pp. 81-93. 
BARTHES, R. (1970): «L'ancienne thétorique», Communications, núm. 16 , pp. 172-223.

BENHABID, S. (1994): «Deliberative Rationality and Models of Democratic Legitimacy», Constellations, núm. 1, pp. 26-52.

Breuer, D., y SChANze, H. (1981): Topik, München, Fink.

Delgado, R. (1991): "Affirmative Action as a Majoritarian Device: Or, Do You Really Want to Be a Role Model?», Michigan Law Review, núm. 89, pp. 1222-1231.

CoHEN, J. (1986); «An Epistemic Conception of Democracy», Ethics, núm. 97, pp. 26-37.

- (1991): «Deliberation and Democratic Legitimacy», en A. Hamlin y Ph. Pettit, The Good Polity, London, Blackwell, pp. 17-34.

Clayton, J. (1993): The Pleasures of Babel, New York, Oxford University Press.

Cox, J. R., y Willard, Ch. (1982): Advances in Argumentation Theory and Research, Carbondale, Southern Illinois University Press.

Enuch, K. (1989): Sprache im Faschismus, Frankfurt, Suhrkamp.

Estunn, D. M. (1993): «Who's Afraid of Deliberative Democracy?", Texas Law Review, núm. 71, pp. 1437-1477.

Gestenberg, O. (1997): Bürgerrechte und deliberative Demokratie, Frankfurt, Suhrkamp.

GethManN, C. F. (1979): Protologik, Frankfurt, Suhrkamp.

- (1980): Theorie des wissenschaftlichen Argumentierens, Frankfurt, Suhrkamp.

Goldman, A. I. (1994): «Argumentation and Social Epistemology», The Journal of Philosophy, pp. 27-49.

Goodn, R, y ReEve, A. (1989): Liberal Neutrality, London, Routledge.

Habermas, J. (1994): «Three Normative Models of Democracy», Constellations, núm. 1, pp. 1-10.
- (1996): Die Einbeziehung des Anderen, Frankfurt, Suhrkamp.

- (1998): «Remarks on Legitimation Throught Human Rights», The Modem Schoolman, pp. 87-100.

Kexes, J. (1993): The Morality of Pluralism, Princeton, Princeton University Press.

- (1992): "The Incompatibility of Liberalism and Pluralism», American Philosophical Quarterly, pp. 141-151.

KLEIN, W. (1980): "Argumentation und Argument', Zeitschrift für Literaturwissenschaft und Logik, núm. 38-39, pp. 9-57.

KOPPERSCHMIDT, J. (1973): Allgemeine Rhetorik, Stuttgart.

- (1981): «Topik und Kritik», en Breuer, D., Schanze, H., Topik, München, Fink, pp. 171-187.

Kopperschmid, J., y Schanze, H. (1985); Argumente-Argumentation, München, Fink.

LeggewIE, C. (1994); Wozu Politikwissenschaft?, Darmstadt, Wissenschaftliche Buchgesellschaft.

López de l.A Vieja, T. (1994): Ética. Procedimientos razonables, Iria Flavia, Novo Seculo.

- (1994): «La presencia de las formas», Revista de Occidente, núm. 154, pp. 103-115.

MaAs, U., y Wunderlich, D. (1972): Pragmatik und sprachliches Handeln, Frankfurt, Athenäum.

Mrlegr, D. (1992): «Deliberative Democracy and Social Choices», Political Studies, pp. 54-67.

Mourse, Ch. (1995): «Politics, Democratic Action, and Solidarity", Inquiry, núm. 38 , pp. 99-108.

Perelman, Ch. (1979): La Lógica juridica y la Nueva retórica, Madrid, Civitas.

Pitkin, H. F., y Shumer, S. (1982): «On Participation», Democracy, núm. 2, pp. 43-54.

Pletr, H. (1977): Rhetorik, München, Fink. Rawls, J. (1993); Political Liberalism, New York, Columbia University Press. 
- (1985): «Justice as Fairness: Political not Metaphysical», Philosophy \& Public Affairs, núm. 14, pp. 223-251.

RA7, J. (1986): The Morality of Freedom, Oxford, Clarendon.

Sunstein, C. (1993): The Partial Constitution, Cambridge, Harvard University Press.

- (1992): «Neutrality in Constitutional Law (With Special Reference to Pornography, Abortion, and Surrogacy)», Columbia Law Review, pp. 1-52.

- (1991): «Preferences and Politics», Philosophy \& Public Affairs, núm. 20, pp. 3-34.

Toulmin, S. (1958): The Uses of Argument, Cambridge, Cambridge University Press.

Trömel-Plötz, S. (1994): Gewalt durch Sprache, Frankfurt, Fischer.

VIEHwEG, Th. (1991): Topica y Filosofia del Derecho, Barcelona, Gedisa.
- (1986): Tópica yjurisprudencia, Madrid, Taurus.

WaltoN, D. N. (1990): «What is Reasoning? What is an Argument? $m$, The Journal of Philosophy, pp. 399-419.

WALZER, M. (1994): Thin and Thick, Notre Dame, University of Notre Dame Press.

WARREN, M. E. (1996): «Deliberative Democracy and Authority", American Political Science Review, núm. 90, pp. 46-60.

Weingerger, O. (1973): «Topik als Plausibilitätsargumentation*, $A R S P$, pp. 17-36.

- (1970): «Wahrheit, Recht und Moral», Rechtstheorie, núm. 2, pp. 129-146.

WeITHMAN, P. J. (1995); «iContractualist Liberalism and Deliberative Democracy?», Philosophy \& Public Affairs, núm. 24, pp. 314-343.

WROBLEWSKI, J. (1989): Sentido y hecho en el Derecho, San Sebastián, Servicio Editorial de la Universidad del País Vasco. 